\title{
MGPDs for calorimeter and muon systems: requirements and first experience in the CALICE test beam
}

\author{
Felix Sefkow ${ }^{1}$ \\ Deutsches Elektronen-Synchrotron DESY \\ Hamburg, Germany \\ E-mail:felix.sefkow@desy.de
}

These proceedings review the application of multi-pixel Geiger mode photo-diodes (MGPDs) for calorimeter and muon systems at the International Linear Collider (ILC). First test beam experience with detectors instrumented with up to 8000 MGPDs is reported. The requirements for MGPD performance parameters and future detector development directions are discussed.

International workshop on new photon-detectors PD07

Kobe University, Kobe, Japan

27-29 June, 2007

\footnotetext{
1 Speaker; representing the CALICE collaboration
} 


\section{Introduction: MGPDs for ILC calorimeter and muon systems}

The International Linear Collider (ILC) [1] is the next big accelerator in high energy physics. While the site and schedule of this global project are still uncertain, the community was requested to have an engineering study and cost estimate for machine and detectors ready by the end of this decade, when first physics results from the Large Hadron Collider (LHC) at CERN are expected and may guide decisions on future facilities. Together with a potential LHC luminosity upgrade programme, the ILC is nowadays the main driver for particle detector R\&D. However, while for the LHC emphasis is on radiation hard sensors and high rate electronics, the ILC demands unprecedented precision and very high numbers of electronic channels.

In electron positron collisions at the ILC, with centre-of-mass energies up to $1 \mathrm{TeV}$, exclusive final states with heavy bosons $(\mathrm{W}, \mathrm{Z}, \mathrm{H})$ are produced. To determine their masses in the resulting multi-jet events requires a jet energy precision which exceeds that of present devices by a factor two or more. According to simulation studies, this can be achieved by reconstructing each particle individually and optimally combining calorimetric and tracking information (particle flow approach, PFLOW) [2].The calorimeters then need to be extremely finely segmented in longitudinal and transverse direction.

With the advent of multi-pixel Geiger mode photo-diodes (MGPDs) [3] this high segmentation can be realized with scintillators at reasonable cost. The millimetre-size MGPDs can operate with moderate bias voltage in high magnetic field and can be mounted right on the scintillator. The light is collected either directly or via embedded wavelength shifting fibres (WLS). This concept is different from existing calorimeters with long fibre readout; it allows integrating not only the photo-sensors but also the front end electronics into the detector volume.
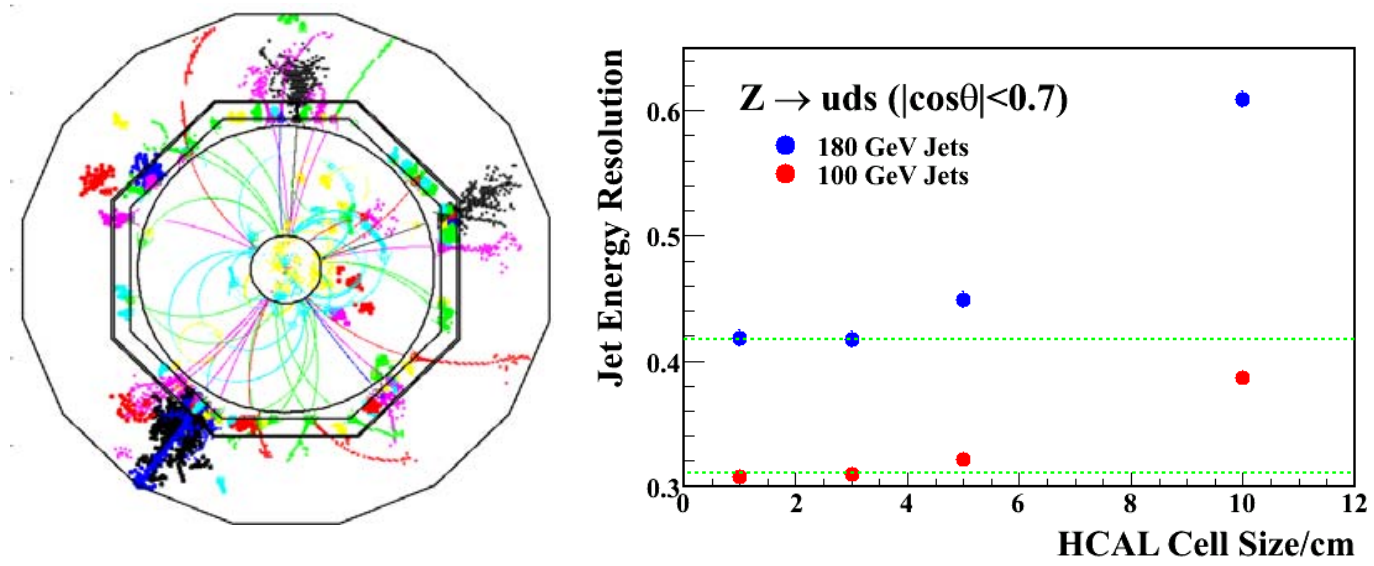

Figure 1 Left: Simulated multi-jet event in a tile HCAL with $3 \times 3 \mathrm{~cm}^{2}$ cells. Right: Dependence of jet energy resolution on scintillator cell size.

The actually required granularity has been studied in detailed detector simulations, using state-of-the art PFLOW reconstruction algorithms [4]. For a scintillator tile steel hadron calorimeter (HCAL) with square tiles a cell size of $3 \mathrm{~cm}$ was found to be optimal, thus confirming earlier studies of two-particle separation. This would correspond to 5 million 
channels in the large detector design. With $1 \mathrm{~cm}$ cells, only minor improvements are seen, at the cost of a tenfold channel count, whereas at $10 \mathrm{~cm}$ the performance is clearly degraded (Figure 1). Thus, while the exact size may undergo some further optimization, the channel count order of magnitude should already be a good estimate.

Similar studies were done for electromagnetic calorimeters (ECAL), where the emphasis is on spatial resolution of compact showers. Here, strips with $1 \mathrm{x} 4 \mathrm{~cm}^{2}$ size were found to be optimal, corresponding to about 10 million channels in total. Muon systems, due to the expected lower occupancy, can afford coarser segmentation, with strips of typically $5 \mathrm{~cm}$ width and several metres length, resulting in "only" order of 50'000 channels.

The scintillator MGPD technology for the ILC competes with alternatives which are also studied in intensive R\&D efforts. For the ECAL, silicon pad sensors offer the advantage of stability, linearity over large dynamic range and the possibility to realize ultra-compact structures with minimal Molière radii, however at the expense of inferior energy resolution and higher cost, driven by the $\sim 3000 \mathrm{~m}^{2}$ of active area to be instrumented with Silicon. For HCAL and muon systems, gaseous devices like resistive plate chambers are proposed, which in principle should offer cost advantages due to simpler readout electronics, even with higher granularity. Operational issues such as stability and calibration will play an important role, apart from the cost, which needs engineering and industrialization studies before it can be reliably assessed.

The R\&D programme follows two parallel streams: test-beam prototypes record data at unprecedented granularity, in order to validate the modelling of hadron shower fine structure, and to test the novel sensor technology at large scale. The design of these calorimeters, however, cannot be extrapolated to full detector system; scaleable solutions, driving sensor and electronics integration to their limit, are being addressed with so-called technical prototypes.

\section{Experience with a large test beam prototype}

The tile HCAL groups $^{1}$ in the international CALICE collaboration [5] have constructed a cubic-metre sized HCAL with 7608 scintillator tiles, individually read out by MGPDs, and tested it in electron and hadron beams at CERN. This prototype is the first device which uses MGPDs on a large scale.

\subsection{Detector structure}

The HCAL is a 38-layer sampling calorimeter made of a plastic-scintillator steel sandwich structure with a lateral dimension of about $1 \mathrm{~m}^{2}$. Each layer (Figure 2) consists of $1.6 \mathrm{~cm}$ thick steel absorber plates and a plane of $0.5 \mathrm{~cm}$ thick plastic scintillator tiles housed in a steel cassette with two $2 \mathrm{~mm}$ thick walls. The total thickness corresponds to $4.5 \lambda$.

The tile sizes vary from $3 \times 3 \mathrm{~cm}^{2}$ for $10 \times 10$ tiles in the centre of the module, to $6 \times 6 \mathrm{~cm}^{2}$ in an intermediate region and $12 \times 12 \mathrm{~cm}^{2}$ in the outer region. Each tile is coupled via a wavelengthshifting (WLS) fibre inserted in a groove to a SiPM via an air gap. The tile faces are covered

${ }^{1}$ DESY, Hamburg U, ICL, UCL London, ITEP, MEPHI, LPI Moscow, Northern Illinois U, LAL Orsay, IPASCR Prague 
with reflector foil; the edges were matted to provide diffuse reflection and suppress optical cross-talk between tiles to below $2 \%$.
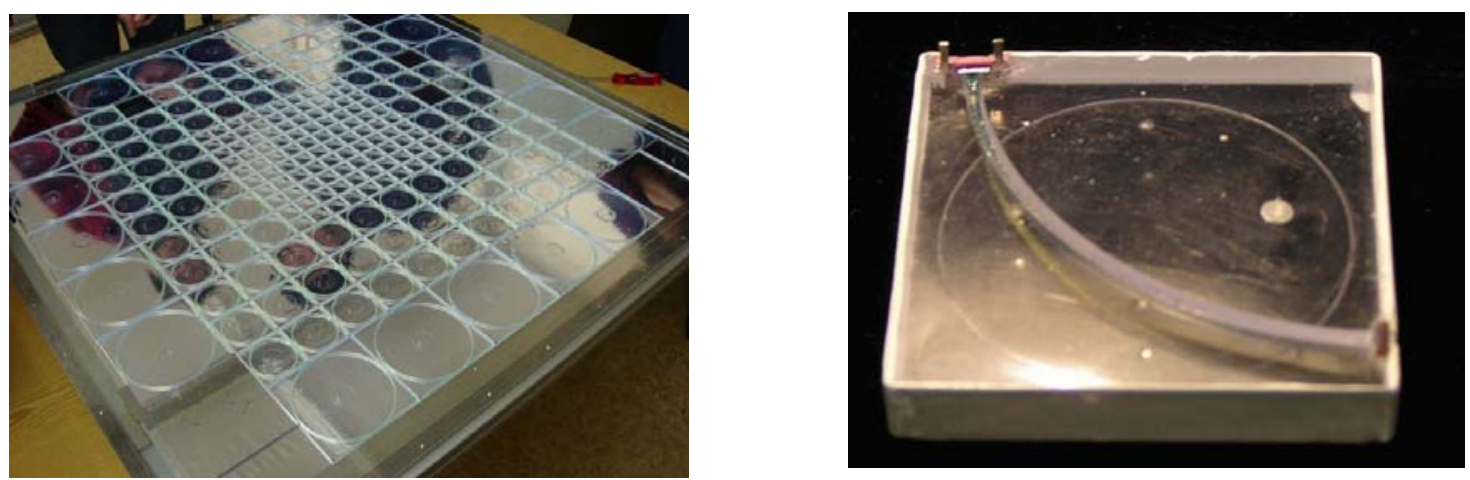

Figure 2 Left: Scintillator tile layer. Right: Scintillator tile $\left(3 \times 3 \mathrm{~cm}^{2}\right)$ with SiPM.

The readout electronics consists of 18 channel front end ASICs [6] with preamplifier shaper and sample-and-hold chain mounted on the cassettes, multiplexed and read-out by an off-detector VME-based ADC system. The ASICs provide a high gain operation mode for autocalibration of the SiPM gain by observation of single photo-electron (p.e.) signals and include DACs for the channel-by-channel adjustment of the SiPM bias voltage.

A versatile and redundant LED calibration system provides light signals up to an equivalent of 200 minimum ionizing particles (MIPs) to each tile. Low light intensities are used for gain calibration; intermediate and high intensities provide PIN diode controlled reference signals for stability monitoring.

\subsection{MGPD production and test}

The MGPDs, here the so-called SiPMs, were developed, manufactured and tested in Russia by the company PULSAR, in collaboration with the MEPHI and ITEP groups. The photosensitive area $\left(1.1 \times 1 \mathrm{~mm}^{2}\right)$ holds 1156 pixels, each with a quenching resistor of a few $\mathrm{M} \Omega$. The detectors are reversely biased with a voltage of $\sim 50 \mathrm{~V}$ and have a gain of $\sim 10^{6}$. Tests have shown that SiPMs are insensitive to magnetic fields up to 4 Tesla and sufficiently radiationhard. For the HCAL prototype, more than 10000 SiPMs have been characterized in an automatic setup, with calibrated LED light. The bias voltage working point was chosen as the one that yields 15 pixels for a MIP-like LED signal. Gain, relative efficiency, dark rate, interpixel cross-talk, noise above a threshold of $1 / 2$ MIP and the non-linear response function of fired pixels $v s$. light intensity over the full dynamic range were measured. The cut on the noise above threshold had the strongest impact on the selection yield and was set to $3 \mathrm{kHz}$. The test results were stored in a data base and are used for calibration and correction procedures. More details about this crucial part of the project are reported in [7].

\subsection{Operational experience and calibration}

The HCAL was assembled and commissioned at DESY, where also an initial calibration of the active layers was obtained in the electron test beam. In 2006, the stack with 23 instrumented layers was exposed to electron and hadron beams of 6-45 GeV and 6-120 GeV, 
respectively, in the $\mathrm{H} 6$ beam line at the CERN SPS. In addition high intensity muon beams were available for calibration. Data taking continued in 2007, with all 38 layers completed. The test beam installation is shown in Figure 3.
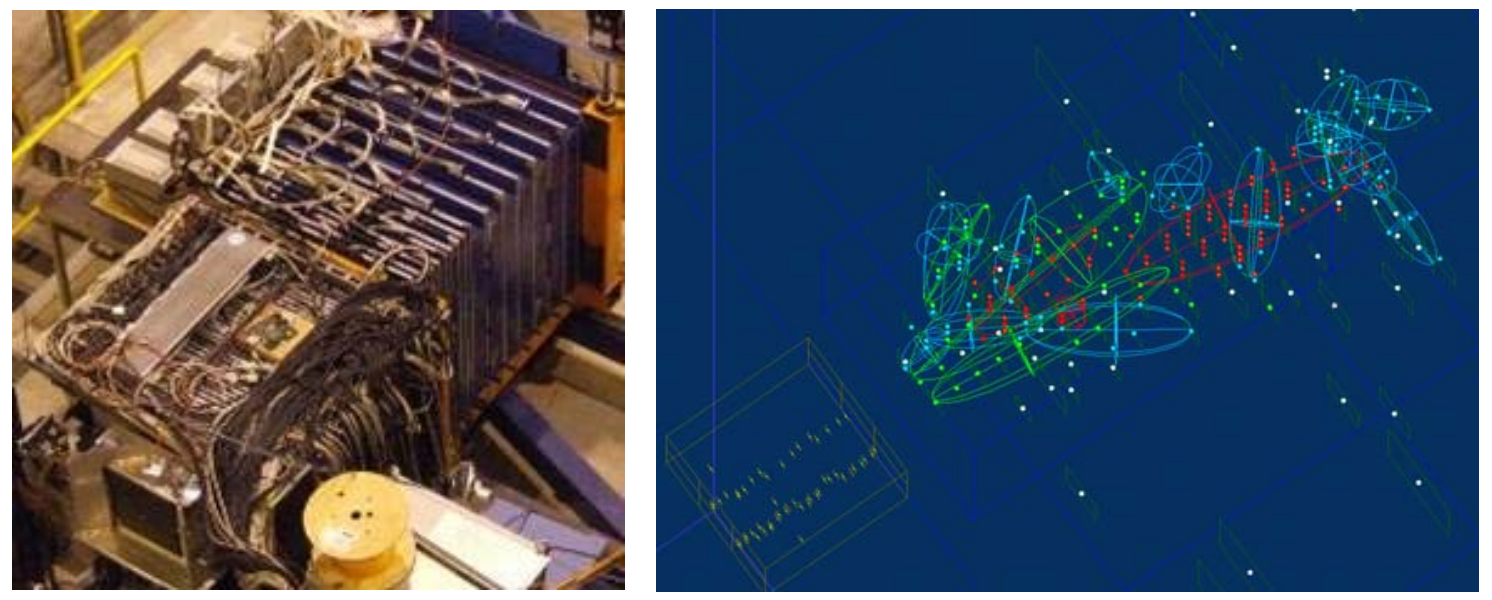

Figure 3 Left: CALICE test beam installation of the HCAL together with the silicon tungsten ECAL (in front) and the scintillator strip tail catcher (behind). Right: Two hadron event seen in ECAL and HCAL, with reconstructed energy clusters.

Detector operation proved to be very stable, with up-times above $90 \%$. The fraction of fully operational channels was $98 \%$, only $1 \%$ suffered from bad soldering, and $1 \%$ of the SiPMs exhibited long discharges. This latter problem was recognized early and cured with improved testing procedures.

All calorimeter cells have been calibrated with muons. The MIP signal $A_{\text {MIP }}$ is used as a scale for the deposited energy and to set the noise suppression threshold of $1 / 2 \mathrm{MIP}$, which yields a MIP hit efficiency of about $95 \%$. The gain $A_{\text {pixel }}$, is measured with low intensity LED light and extracted from the single p.e. peak separation. It is needed for non-linearity corrections and is also used to verify operation at the correct bias voltage setting by checking that the light yield $A_{\text {MIP }} / A_{\text {pixel }}$ corresponds to 15 pixels per MIP on average. The noise hit occupancy in the detector is then about $10^{-3}$, as expected from the test bench, and corresponds to $\sim 0.1 \mathrm{GeV}$ deposited energy on the electromagnetic (e.m.) energy scale.

The visible energy per cell in units of MIPs is obtained from the formula

$$
E[\mathrm{MIP}]=A / A_{\mathrm{MIP}} F\left(N_{\text {pixel }}\right) \text { with } \quad N_{\text {pixel }}=A / A_{\text {pixel }}
$$

where $A$ are amplitudes measured in ADC counts. The non-linearity correction $F$ depends only on the amplitude in units of pixels and is 1 for small amplitudes (all scale factors are absorbed in the MIP calibration factor). The function $F$ is the inverse of the normalized response function and can be approximated as $F=-N / N_{\text {pixel }} * \log \left(1-N_{\text {pixel }} / N\right)$ for a total of $N$ active pixels on the SiPM. In practice $F$ is obtained from the test bench measurements. The conversion from MIPs to deposited energy depends on the incident particle type and is taken from simulations or using the known beam energy as reference.

MIP and pixel scale are subject to temperature variations of a few percent per Kelvin. The redundant monitoring system offers various possibilities for correcting these effects, using gain, LED reference signals or direct temperature measurement. The procedures are under 
development and not yet applied for the first analyses which use data from sufficiently stable periods.

\section{$2.4 \quad$ First results}

The imaging capabilities of the detector became immediately obvious already on the online event display. Figure 3 shows a (rare) event with two hadrons in ECAL and HCAL. First, there is a rich substructure visible which can be exploited for energy weighting procedures, by proper classification of sub-clusters as of hadronic or electromagnetic type, using topology and energy information. Second, it appears possible to disentangle the independent development of the two partially overlapping showers. Since the hit occupancy in the high granularity is small, this can be studied quantitatively using event overlay techniques.

In order to validate the understanding of the detector and its calibration, data have been taken with electrons impinging on the HCAL directly. Figure 4 shows the response as a function of the beam energy. Clearly the linearity is not perfect yet with the preliminary correction procedures, but it is sufficient for the analysis of hadron data. The hadronic energy resolution (Figure 4) is found to be within the range of expectations. In particular there is no indication of a large constant term, which would otherwise signal problems with hardware or calibration.

Due to the not yet complete instrumentation in 2006 the hadron data still cover a limited energy range. This will be extended with data taken in 2007 at CERN and 2008 at FNAL. They also feature tests with inclined beam incidence, and with identified protons.
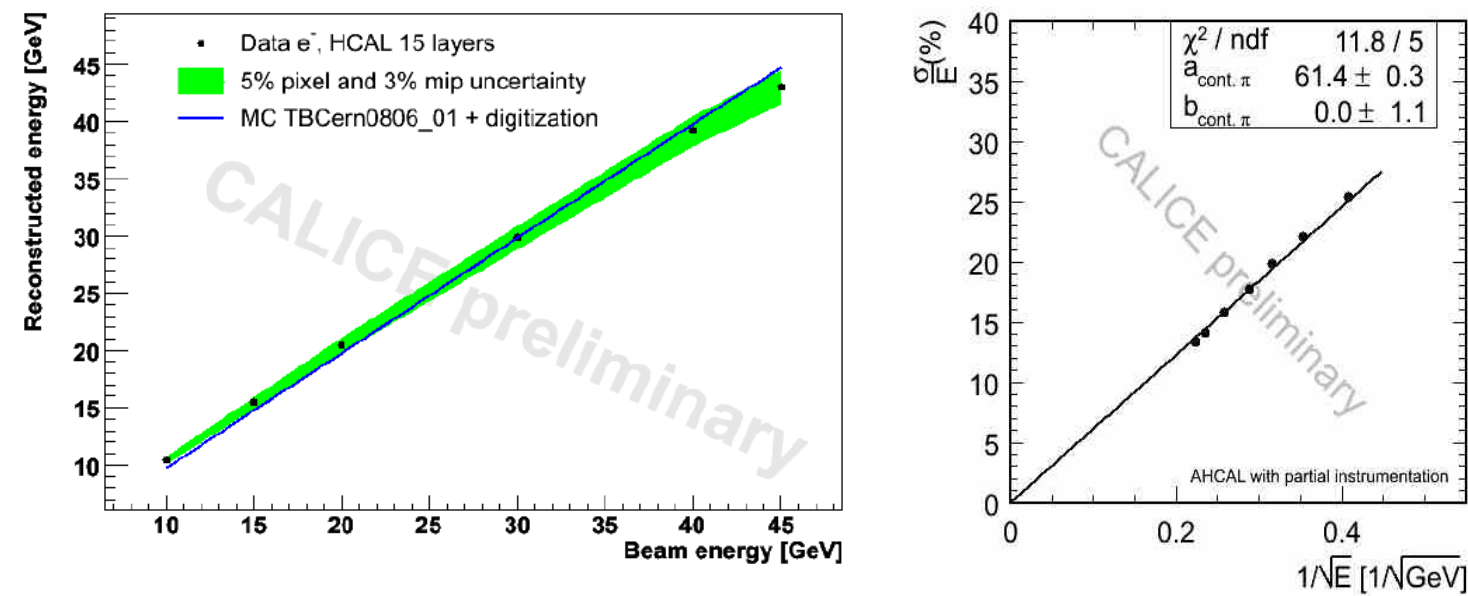

Figure 4: Left: HCAL response to electrons, vs. beam energy. Right: HCAL resolution on electromagnetic scale, vs. $1 / \sqrt{ } E$ ( $E$ is the beam energy).

\section{Future directions and requirements}

In the next years, the technology needs to be developed and optimized further, in order to proceed from the pioneering test beam experience towards a realistic and cost-effective proposal for ILC detectors. This challenge goes beyond a straight-forward engineering effort, since no example for a scintillator-based detector with embedded photo-sensors and front end electronics exists so far. To meet the goal, further R\&D is needed both for the active readout layer electromechanical design, which must integrate the microelectronics readout chips and minimize the cost-sensitive thickness of the readout gap, as well as for the optimization of the scintillator 
MGPD system. Operational experience and analysis results from the ongoing test beam programme are expected to significantly influence this development.

\subsection{Mechanical structure with integrated front end electronics}

A possible solution for the layout of an HCAL barrel module with integrated electronics is sketched in Figure 5. The active layers are subdivided into PCBs of manageable size; here each hosts 4 front-end ASICs which each digitize the signals of 36 MGPDs. Data are collected at the layer end and further concentrated at module level before being sent to the off-detector DAQ. The ILC beam time structure with $1 \mathrm{~ms}$ long bunch trains separated by $200 \mathrm{~ms}$ gaps permits to minimize power consumption and thus heat dissipation by switching the power supply in the ASICs such that an average of $40 \mu \mathrm{W}$ per channel generate negligible temperature gradients in the structure, and no internal cooing is needed.

The cross-section illustrates an idea to eliminate the optical fibre system for calibration light distribution by embedding tiny surface-mounted LEDs in the PCBs. This is possible since thanks to the auto-calibrating features of the MGPDs the stability of the light source does not need to be monitored.

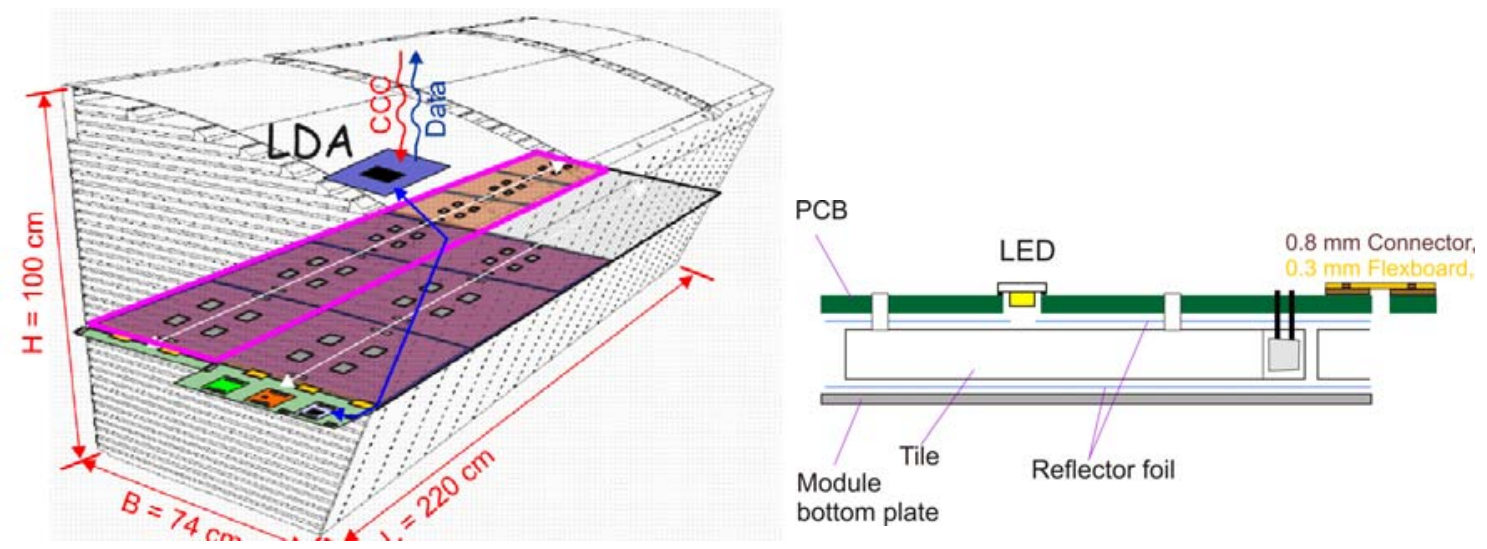

Figure 5 Left: Layout of an HCAL barrel module with embedded read-out electronics. Right: Cross section with details.

For the integration of the MGPD, the PCB and the scintillator different approaches are being followed. The first is to produce and test MGPD scintillator systems as units, which are then coupled to the electronics PCB. The second is to integrate the MGPD first into the PCB and later to couple bare tiles to the fully instrumented PCB. The first option, which was also followed for the test beam prototype, minimizes the risk of instabilities in the optical coupling and allows easy and early quality control independent of the final electronics and its schedule. The advantage of the second is the possibility to include the MGPD mounting in the automated SMD technology. While the first option works with or without WLS fibre, the second requires direct coupling of blue-sensitive MGPDs to the tiles.

Direct coupling of MGPDs to scintillator strips or tiles has been studied intensively by various groups, using different sensor types [7]. The recently available MPPC with 1600 pixels, for example, would meet the requirements for hadron calorimetry, in terms of efficiency, noise, and dynamic range; however it leaves no headroom for thinning the scintillator tiles. While direct coupling is an elegant design providing new and simplified options for assembly, it has to 
be noted that the omission of the WLS fibre introduces considerable non-uniformities in the response of scintillator tiles, which increase with reduced tile thickness. For strips, sufficient uniformity has been achieved with or without fibre.

\subsection{Critical parameters of MGPDs for detector optimization}

The requirements for MGPD parameters have their origin in different aspects of the detector system performance. The emphasis is slightly varying between ECAL, with strong demands on dynamic range, and HCAL and muons systems, where the efficiency for small signals and low noise occupancy are more critical.

A small MGPD dark rate helps to keep the noise above a given threshold low. The ultimate limitation, however, comes from the requirement to observe single p.e. peaks for gain calibration and non-linearity correction. For this purpose, the probability of noise pile-up must be kept below $\sim 10 \%$. With finite front end electronics shaping times of typically $50 \mathrm{~ns}$, this translates into an upper limit of $2 \mathrm{MHz}$ for the dark rate per device.

Radiation hardness has been more extensively discussed in other presentations, e.g. [7]. At the ILC, this is not an issue, except in detector regions very near the beam pipe, where neutron fluxes above $10^{10} / \mathrm{cm}^{2}$ can occur. MGPDs can also operate in harsher environmental conditions, if higher dark rates can be tolerated, e.g. if single p.e. peaks do not need to be observed.

Even with lowest noise rates - or excellent signal over noise ratio -, statistical fluctuations in the number of detected p.e. introduce an absolute lower limit on the light yield to be achieved with a given scintillator for a MIP, and thus on the efficiency of the photo-sensor. In order to detect a signal larger than 1 p.e. with Poisson probability above 95\%, the mean light yield must be $>4.5$ p.e., if Landau fluctuations are also taken into account. In practice, additional margins are needed for light yield variations, threshold uncertainties and electronic noise, such that about 8 p.e. are a safe limit..

The inter-pixel cross-talk determines how steeply the noise rate falls with threshold. The noise above threshold $v_{\mathrm{T}}$ depends on all three quantities: dark rate $v$, cross-talk $X$ and efficiency via the threshold $T$ (in p.e.) which can be applied (typically at $1 / 2$ MIP). As a rule of thumb, one may estimate $v_{\mathrm{T}}=v X^{T-0.5}$. The occupancy or random hit probability $P$ is proportional to the length $\Delta \mathrm{t}$ of the gate during which the electronics integrates the signal, $P=v_{\mathrm{T}} \Delta \mathrm{t}$. Thus, in principle, it can be minimized with fast electronics. However, for the reconstruction of hadron showers one aims at collecting also signals delayed by nuclear de-excitation, so the gate should remain open for the entire bunch crossing interval of $300 \mathrm{~ns}$. An occupancy limitation of $10^{-4}$ then translates into a noise limit of $v_{\mathrm{T}}<300 \mathrm{~Hz}$. This limitation originates from readout bandwidth considerations and from the need to discriminate isolated neutron hits from spurious electronic background.

Experience with the test beam prototype has shown that after precise adjustment the occupancy was small enough for the envisaged physics studies. However, the ultimate ILC goal could not yet be reached, even though the selection criteria were carefully optimized. So, improvements or leeway for easier operation would be desirable. More light would help, but would result in a reduction of dynamic range. Lower dark rate would be helpful, too, but enters only linearly. Larger sensor area, which is possible as long as the dark rate remains below the 
absolute limit, is an attractive option, since it would permit to raise thresholds and would still offer larger dynamic range. The most drastic improvements evidently result from reducing interpixel cross-talk, which is actively being worked on by sensor developers.

The required dynamic range, finally, is still under study. For muon detection, this is not critical. For the HCAL, the performance of presently available sensors appears to be just adequate, yet more quantitative studies from the test beam data should be awaited. For the ECAL, demands are much more aggressive. For $50 \mathrm{GeV}$ e.m. showers, single channel amplitudes up to 1000 MIPs need to be recorded with adequate precision, which in practice limits non-linearity corrections to below $50 \%$. For a light yield of 10 pixels / MIP, a sensor which saturates at 10000 pixels would be needed. The effectively available number of pixels depends on the ratio of sensor recovery time and light pulse duration: a fast sensor on a slow pulse can fire the same pixel several times. For the MPPC-1600, with fast recovery times of about $4 \mathrm{~ns}$, the signal did not even saturate at amplitudes above 5000 pixels [9]. If this effective increase in dynamic range is to be exploited, it requires very precise control of the non-linearity and of the time structure of calibration signals.

\section{Summary and conclusion}

The novel multi-pixel Geiger mode photo-diodes open revolutionary detector design options with highly segmented scintillators, which meet the ILC performance goals. A prototype with 8000 channels has been built and successfully operated in the CERN test beam. The results will be used to test the simulations and guide the further optimization of the detector concept. In the next years, more R\&D should be done to further consolidate and optimize the technology, and to proceed towards a realistic detector design. In conclusion, the proof-ofprinciple of the MGPD technology looks very promising. Yet, the quest for best MGPD performance and competitiveness goes on.

\section{References}

[1] J. Brau et al., International Linear Collider reference design report, Vol 1: Executive summary, ILCREPORT-2007-001 (2007).

[2] J. Brau et al., International Linear Collider reference design report, Vol 4: Detector, ibid.

[3] D. Renker, these proceedings, and references therein.

[4] M. Thomson, presentation at the 2007 International Linear Collider Workshop (LCWS07 and ILC07), Hamburg, Germany, 30 May - 3 Jun 2007.

[5] CALICE Collaboration (C. Adloff et al.), CALICE Report to the Calorimeter R\&D Review Panel, ILC-DET-2007-024, arXiv:0707.1245.

[6] J. Fleury, these proceedings.

[7] E. Tarkovsky, these proceedings.

[8] N. D’Ascenzo, these proceedings.

[9] S. Uozumi, these proceedings. 